\title{
The Essence of XML (preliminary version)
}

\author{
Jérôme Siméon ${ }^{1}$ and Philip Wadler ${ }^{2}$ \\ 1 Bell Laboratories, Murray Hill, NJ 07974, USA \\ simeon@research.bell-labs.com \\ 2 Avaya Labs Research, Basking Ridge, NJ 07920, USA \\ wadler@avaya.com
}

\begin{abstract}
The World-Wide Web Consortium (W3C) promotes XML and related standards, including XML Schema, XQuery, and XPath. This paper describes a formalization XML Schema. A formal semantics based on these ideas is part of the official XQuery and XPath specification, one of the first uses of formal methods by a standards body. XML Schema features both named and structural types, with structure based on tree grammars. While structural types and matching have been studied in other work (notably XDuce, Relax NG, and previous formalizations of XML Schema), this is the first work to study the relation between named types and structural types, and the relation between matching and validation.
\end{abstract}

\section{Introduction}

There are a number of type systems for XML, including: DTDs, part of the original W3C recommendation defining XML [1]; XML Schema, a W3C recommendation which supersedes DTDs [13]; Relax NG, an Oasis standard [5]; Relax [10] and TREX [4], two ancestors of Relax NG; and the type systems of XDuce [8] and YATL [6]. All of these take a structural approach to typing, with the exception of XML Schema, which takes a named approach. (Another possible exception is DTDs, which are so restricted that the named and structural approaches might be considered to coincide.)

The W3C is responsible for three programming languages connected with XML: XSLT, a language for stylesheets [3,9]; XQuery, an analogue of SQL for XML data [15]; and XPath, the common core of XSLT and XQuery, which is jointly managed by the working groups responsible for the other two languages [14]. All three of these are functional languages. XSLT 1.0 and XPath 1.0 became recommendations in November 1999 - they are untyped. XML Schema 1.0 became a recommendation in May 2001. XSLT 2.0, XQuery 1.0, and XPath 2.0 are currently being designed - they have type systems based on XML Schema.

This paper presents a formalization of XML Schema, developed in conjunction with the XQuery and XPath working groups. The paper presents a simplified version, treating the essential constructs. The full version is being developed as 
part of the XQuery and XPath Formal Semantics [16], one of the first industrial specifications to exploit formal methods. The full version treats not just XML Schema, but also the dynamic and static semantics of the XQuery and XPath.

Formal methods are particularly helpful for typing - the only complete description of the static type system of XQuery and XPath is in the formal specification. However, keeping two specifications in sync has not always been easy.

An earlier formal specification of XML Schema [2] was influenced by XDuce [8]; it ignored the named aspects of Schema and took a purely structural approach. The specification of Relax NG [5] also uses formal methods, and also is purely structural; it was influenced by the earlier work on XML Schema [2].

Matching and validation. Types in XML differ in some ways from types as used elsewhere in computing. Traditionally, a value matches a type - given a value and a type, either the value belongs to the type or it does not. In XML, a value validates against a type - given an (external) value and a type, validation produces an (internal) value or it fails.

For instance, consider the following XML Schema.

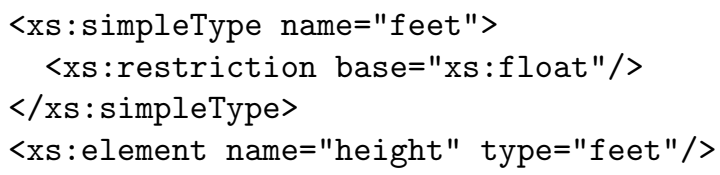

In our type system, this is written as follows.

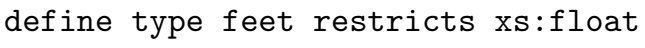

Now consider the following XML document.

$$
\text { <height>10023</height> }
$$

In our model, before validation this is represented as follows.

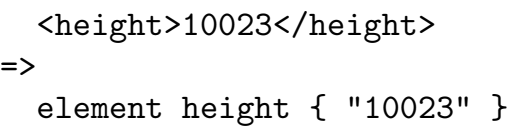

And after validation it is represent as follows.

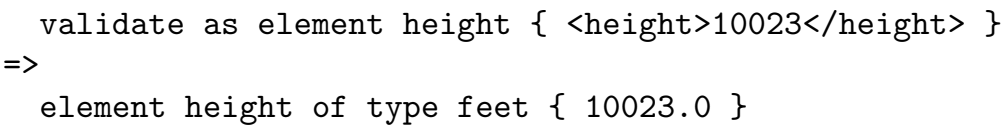

Validation has annotated the element with its type, and converted the text "10023" into the corresponding floating point number 10023.0.

Our model provides both validation and matching. Validation attaches types to XML data. Unvalidated data may not match against a type. The following does not hold. 


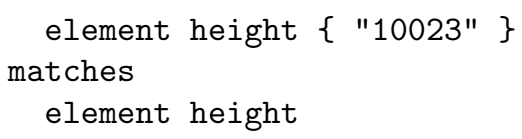

After validation, matching succeeds. The following does hold.

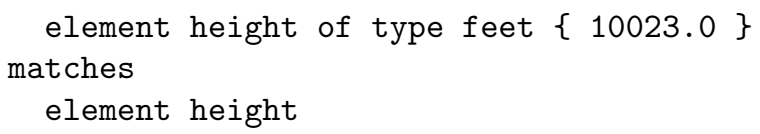

The inverse of validation is type erasure.

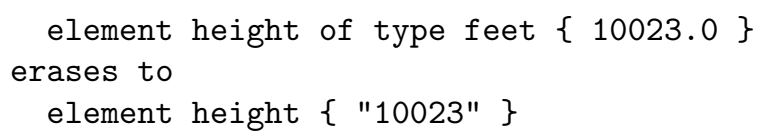

The following theorem characterizes validation in terms of matching and erasure.

Theorem 1. We have that

validate as Type $\{$ UntypedValue $\} \Rightarrow$ Value

if and only if

Value matches Type

and

Value erases to UntypedValue

Perhaps this theorem looks obvious, but if so let us assure you that it was not obvious to us when we began. It took some time to come to this formulation, and some tricky adjustments were required to ensure that holds.

One trick is that we model validation and erasure by relations, not functions. Naively, one might expect validation to be a partial function and erasure to be a function. That is, for a given type each untyped value validates to yield at most one typed value, and each typed values erases to one untyped value. One subtlety of the system presented here is that validation and erasure are modeled by relations. For example, the strings "10023" and "10023.0" both validate to yield the float 10023.0, and hence we also have that the float erases to yield either string.

Shortcomings of XML and Schema. Our aim is to model XML and Schema as they exist - we do not claim that these are the best possible designs. Indeed, we would argue that XML and Schema have several shortcomings.

First, we would argue that a data representation should explicitly distinguish, say, integers from strings, rather than to infer which is which by validation against a Schema. (This is one of the many ways in which Lisp S-expressions are superior to XML.)

Second, while derivation by extension in Schema superficially resembles subclassing in object-oriented programming, in fact there are profound differences. One can typecheck code for a class without knowing all subclasses of that class 
(this supports separate compilation), but one cannot typecheck against a Schema type without knowing all types that derive by extension from that class (and hence separate compilation is problematic).

Nonetheless, XML and Schema are widely used standards, and there is value in modeling these standards. In particular, such models may: (i) improve our understanding of exactly what is mandated by the standard, (ii) help implementors create conforming implementations, and (iii) suggest how to improve the standards.

Relation of our model to Schema. Schema is a large and complex standard. In this paper, we attempt to model only the most essential features. These include: simple types and complex types; named and anonymous types; global and local elements; atomic, list, and union simple types; derivation by restriction; and derivation by extension. We model only two primitive datatypes, xs:float and xs:string, while Schema has nineteen primitive datatypes.

Many features of Schema that are omitted here are dealt with in the formal semantics for XQuery [16]. These include: namespaces; attributes; all groups (interleaving); text nodes; mixed content; substitution groups; xsi:nil attributes; and xsi:type attributes. There are other features of Schema that are not yet dealt with in the full formal semantics, but which we hope to model in future. These include: abstract types; default and fixed values; skip, lax, and strict wildcards; and facets of simple types.

Schema is normally written in an XML notation, but here we use a notation that is more readable and compact. The mapping of XML notation into our notation is described in the XQuery formal semantics.

There are a few aspects in which our treatment diverges from Schema. First, we permit ambiguous content models, while Schema does not. We do this because it makes our model simpler, and because ambiguity is important to support type checking, as discussed in Section 9. Second, we permit one type to be a restriction of another whenever the set of values belonging to the first type is included in the set of values belonging to the second, while Schema imposes ad hoc syntactic constraints. Again, we do this because it makes our model simpler, and because our more general model better supports type checking. Third, we only support the occurrence operators ?, +, and *, while Schema supports arbitrary counts for minimum and maximum occurrences. This is because arbitrary counts may lead to a blow-up in the size of the finite-state automata we use to check when one type is included in another.

\section{XML Schema by example}

XML Schema supports a wide range of features. These include simple types and complex types, anonymous types, global and local declarations, derivation by restriction, and derivation by extension.

Simple and complex types. Here are declarations for two elements of simple type, one element with a complex type, and one complex type. 


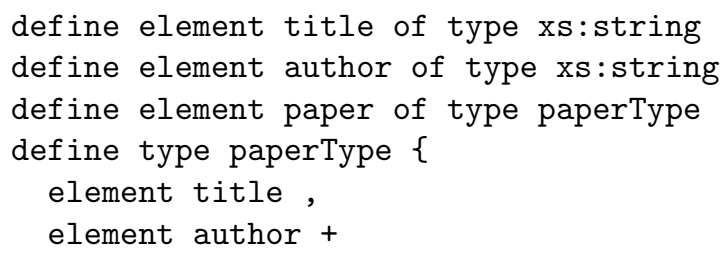

Schema specifies nineteen primitive simple type types, including xs: string and xs:float.

A type declaration associates a name and a structure. The structure of a complex type is a regular expression over elements. As usual, , denotes sequence, I denotes alternation, ? denotes an optional occurrence, + denotes one or more occurrences, and $*$ denotes zero or more occurrences.

Validating annotates each element with its type.

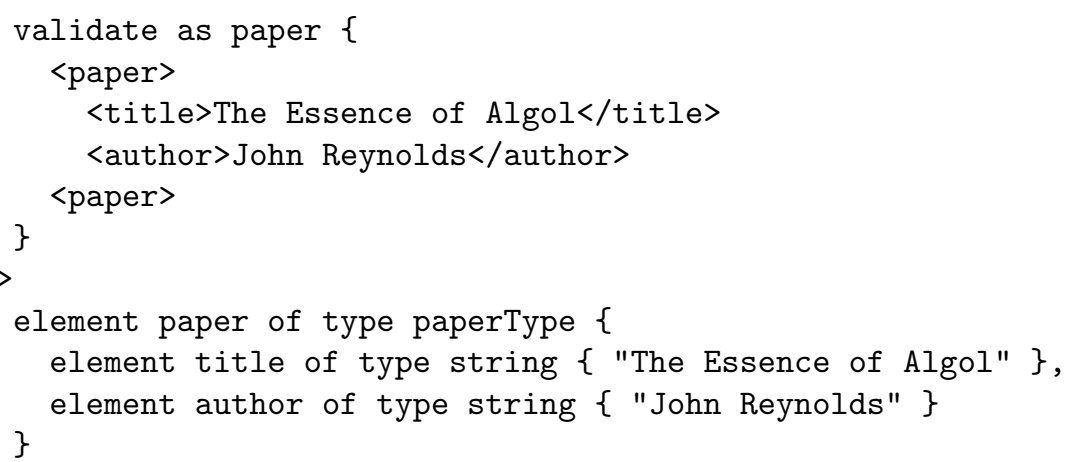

Anonymous types. Instead of naming a type, it can defined in place without a name. Here is the paper element with its type expanded in place.

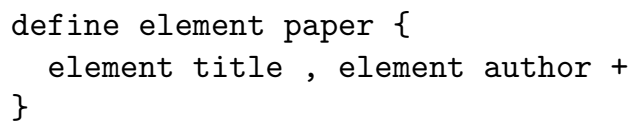

Validating now yields the following result.

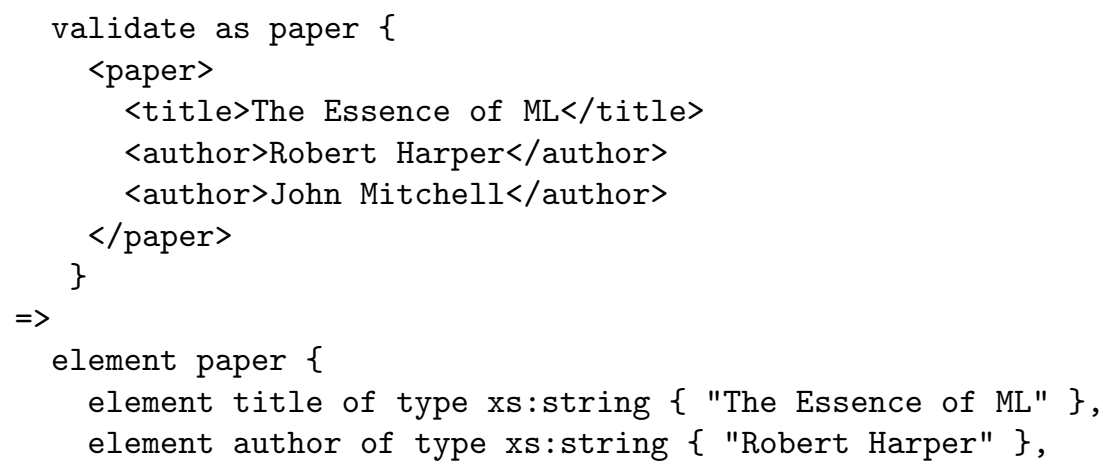




\section{\}}

element author of type xs:string \{ John Mitchell" \}

Now the paper element has no type annotation, because there is no type name to annotate it with. The other elements still have type annotations.

Global and local declarations. Similarly, one may include an element declaration in place. Here is the paper element with the nested elements expanded in place.

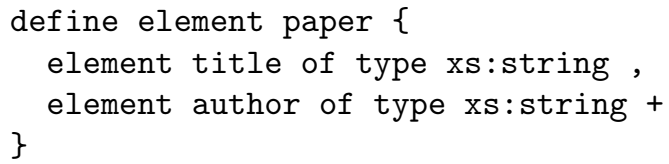

Here the paper is declared globally, while title and author are declared locally. In this case, validation proceeds exactly as before.

Allowing local declarations increases expressiveness, because now it is possible for elements with the same name to be assigned different types in different places; see $[11,7]$. An example of such a definition appears later.

Atomic, list, and union types. Every simple type is an atomic type, a list type, or a union type. The atomic types are the nineteen primitive types of Schema, such as xs:string and xs:float, and the types derived from them. List types are formed using the occurrence operators ?, +, and $*$, taken from regular expressions. Union types are formed using the alternation operator I, also taken from regular expressions.

Here is an example of a list type.

element floats $\{$ xs:float + \}

In XML notation, lists are written space-separated.

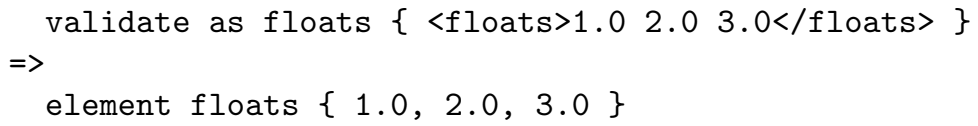

Some types may be ambiguous. XML Schema specifies how to resolve this ambiguity: every space is taken as a list separator, and in case of a union the first alternative that works is chosen.

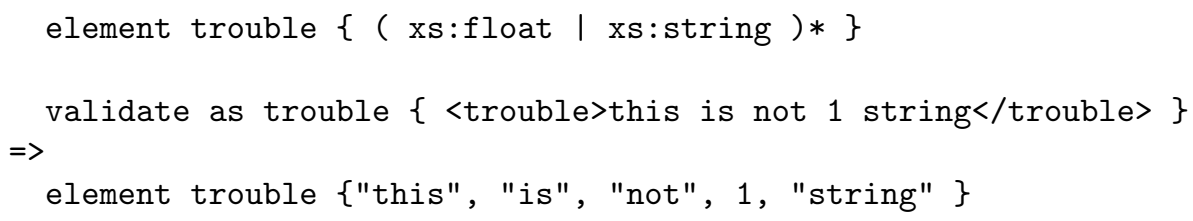

Ambiguous types can be problematic; this will be further discussed in Section 9 . 
Derivation by restriction on simple types. New simple types may be derived by restriction.

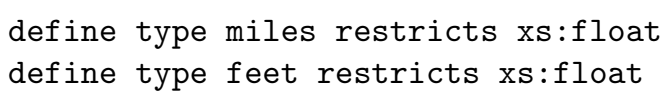

Here is an example, with two height elements have different types.

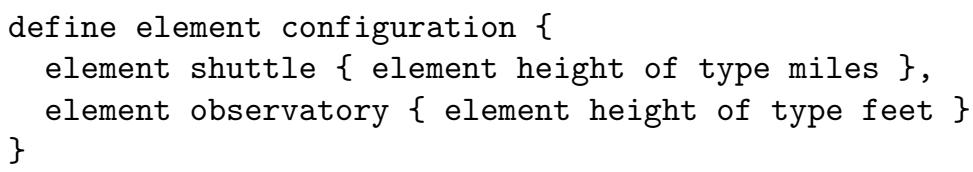

We have the miles and feet are both subtypes of xs:float, but neither is a subtype of the other. The following function definition is legal.

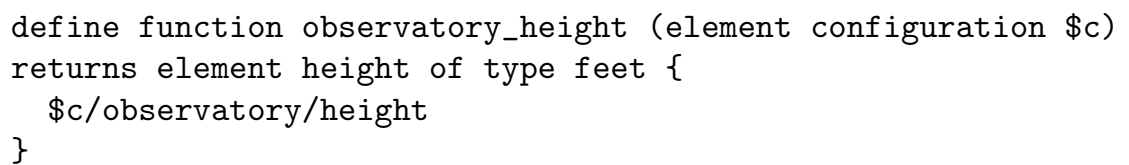

It would still be legal if feet were replaced by xs:float, but not if it were replaced by miles. In this example, element configuration is the type of the formal parameter \$c, the XPath expression \$c/observatory/height extracts the observatory child of the configuration element, and then extracts the height child of the observatory element.

Derivation by restriction on complex types. New complex types may also be derived by restriction. The following example is a simplified form of the information that may occur in a bibliographic database, such as that used by Bib-TeX.

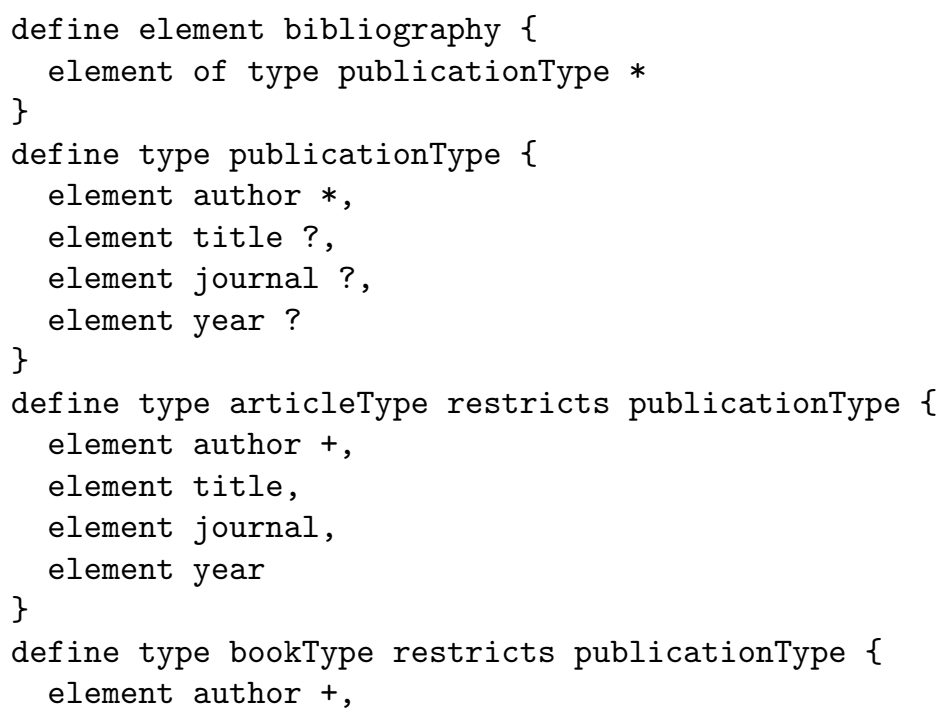




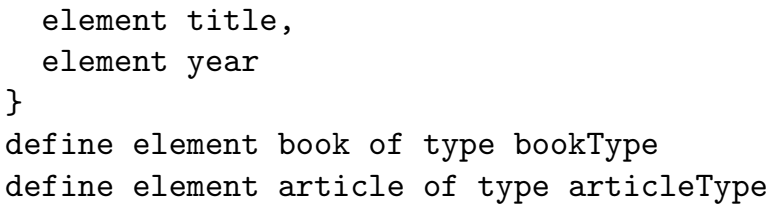

Here a publication may have any number of authors, a mandatory title, and a optional journal, and year. An article must have at least one author, and a mandatory title, journal, and year. A book must have at least one author, a mandatory title and year, and no journal.

Derivation by restriction declares a relationship between two types. This relation depends on both names and structures, in the sense that one name may be derived by restriction from another name only if every value that matches the structure of the first also matches the structure of the second.

When one type is derived from another by restriction, it is fine to pass the restricted type where the base type is expected. For example, consider the following function.

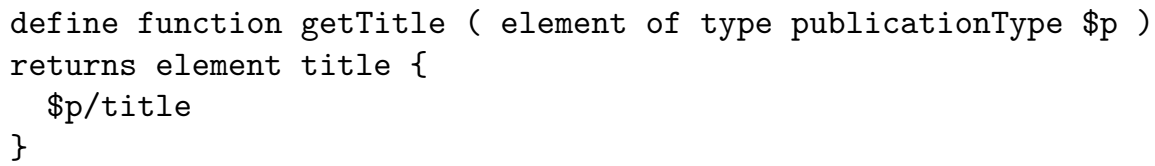

Here it is acceptable to pass either an article or book element to the function getTitle().

There is a type xs:anyType at the root of the type hierarchy. If a type definition does not specify otherwise, it is considered a restriction of xs : anyType.

Derivation by extension. New complex types may also be derived by extension.

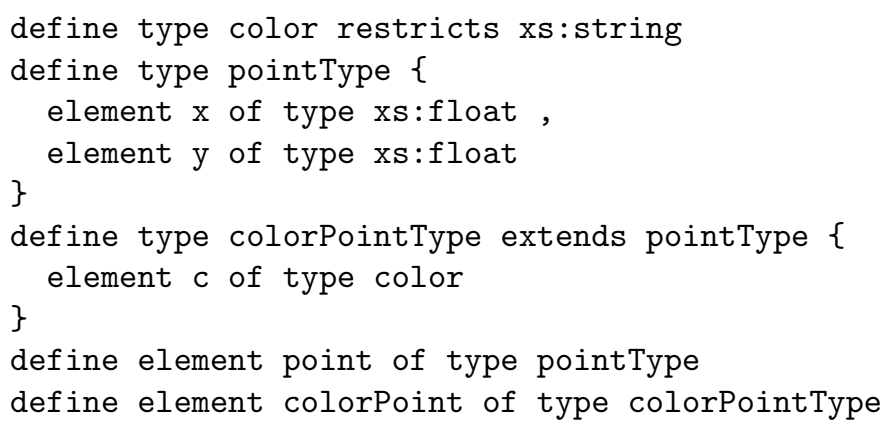

When one type restricts another, one must check that the proper relation holds between the types. When one type extends another, the relation holds automatically, since values of the new type are defined to consist of the concatenation of values of the base type with values of the extension.

Again, when one type is derived from another by extension, it is fine to pass the extended type where the base type is expected. Unlike with restriction, this can lead to surprising consequences. Consider the following. 


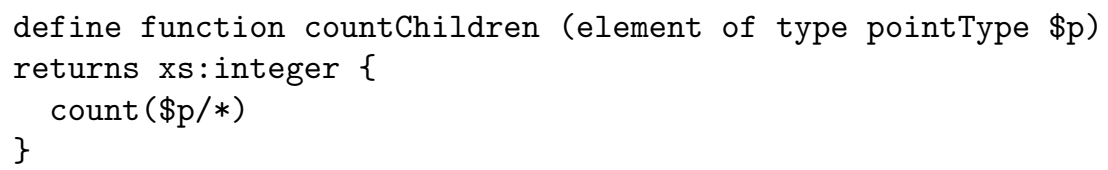

This function counts the number of children of the element $\$ \mathrm{p}$, which will be 2 or 3, depending on whether $\$ \mathrm{p}$ is an element of type point or colorPoint.

In XQuery, type checking requires that one knows all the types that can be derived from a given type - the type is then treated as the union of all types that can be derived from it. Types derived by restriction add nothing new to this union, but types derived by extension do. This "closed world" approach - that type checking requires knowing all the types derived from a type is quite different from the "open world" approach used in many object-oriented languages — where one can type-check a class without knowing all its subclasses.

In an object-oriented language, one might expect that if an element of type colorPoint is passed to this function, then the $\mathrm{x}$ and $\mathrm{y}$ elements would be visible but the c element would not be visible. Could the XQuery design adhere better to the object-oriented expectation? It is not obvious how to do so. For instance, consider the above function when pointType is replaced by xs:anyType.

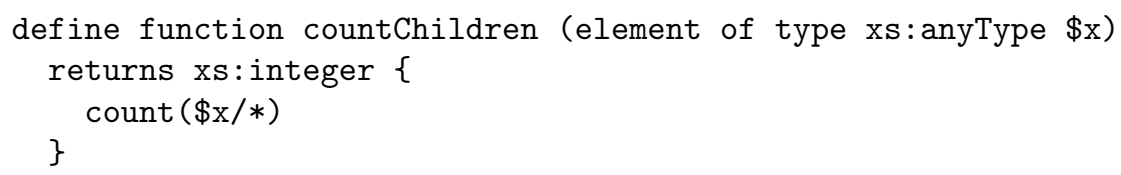

Here it seems natural to count all the children, while an object-oriented interpretation might suggest counting none of the children, since xs:anyType is the root of the type hierarchy.

\section{$3 \quad$ Values and types}

This section describes values and types. For brevity, we consider only 2 atomic types: String and Float.

\subsection{Values}

A value is a sequence of zero or more items. An item is either an element or an atomic value. An atomic value is a string or a float. Elements are optionally annotated with their type. An element with no type annotation is the same as an element with the type annotation xs:anyType.

$\begin{array}{lll}\text { Value } & := & () \\ & \mid & \text { Item }(, \text { Item)* } \\ \text { Item } & :=\text { element ElementName Annotation? \{ Value }\} \\ & & \text { Atomic Value } \\ \text { AtomicValue } & := & \text { String } \mid \text { Float } \\ \text { Annotation } & ::= & \text { of type TypeName }\end{array}$


We also write Value $_{1}$, Value 2 for the concatenation of two values.

An untyped value is a sequence of zero or more untyped items. An untyped item is either an element without type annotation or a string. Untyped values are used to described XML documents before validation. Every untyped value is a value.

$$
\begin{array}{lcl}
\text { UntypedValue } & := & () \\
& \mid & \text { UntypedItem }(, \text { UntypedItem }) * \\
\text { UntypedItem } & := & \text { element ElementName \{ UntypedValue }\} \\
& \mid & \text { String }
\end{array}
$$

A simple value consists of a sequence of zero or more atomic values. Every simple value is a value.

$$
\begin{array}{rcl}
\text { SimpleValue } & := & () \\
& \mid & \text { Atomic Value }(, \text { Atomic Value }) *
\end{array}
$$

Here are some examples of values.

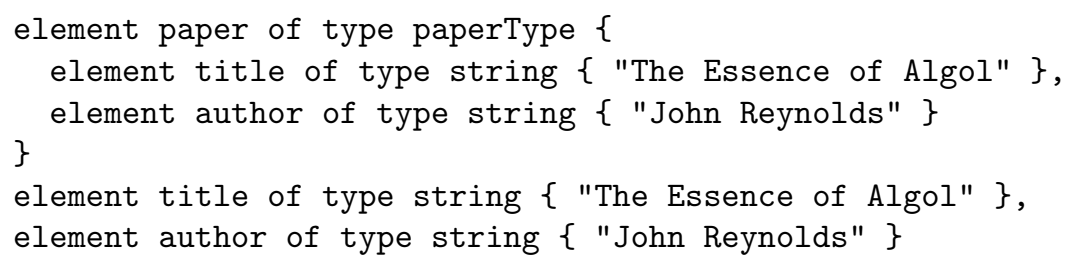

Here are some examples of untyped values.

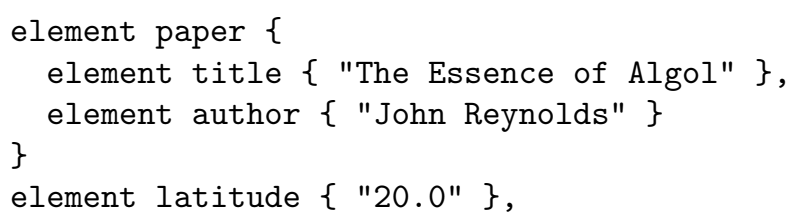

Here are some examples of simple values.

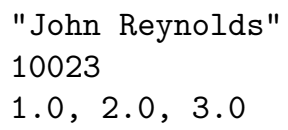

\section{$3.2 \quad$ Types}

Types are modeled on regular tree grammars $[12,7]$. A type is either an item type, the empty sequence $(())$, or composed by sequence (,), choice (I), or multiple occurrence - either optional (?), one or more $(+)$, or zero or more $(*)$.

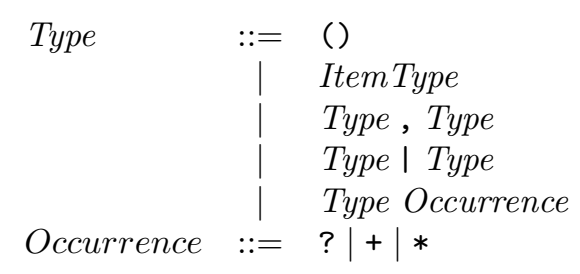


An item type is an element type or an atomic type. Atomic types are specified by name; these names include xs:string and xs:float.

$$
\begin{array}{lll}
\text { ItemType } & := & \text { ElementType } \\
& \mid & \text { AtomicTypeN } \\
\text { AtomicTypeName } & := & \text { TypeName }
\end{array}
$$

An element type gives an optional name and an optional type specifier. A name alone refers to a global declaration. A name with a type specifier is a local declaration. A type specifier alone is a local declaration that matches any name. The word "element" alone refers to any element.

\section{ElementType $:=$ element ElementName? TypeSpecifier?}

A type specifier either references a global type, or defines a type by derivation. A type derivation either restricts an atomic type, or restricts a named type to a given type, or extends a named type by a given type.

$$
\begin{array}{lll}
\text { Item Type } & :=\begin{array}{l}
\text { TypeReference } \\
\text { TypeDerivation } \\
\text { TypeReference }
\end{array} \\
\text { TypeDerivation } & \begin{array}{l}
\text { of type TypeName } \\
\text { restricts AtomicTypeName } \\
\text { restricts TypeName \{ Type }\} \\
\text { extends TypeName }\{\text { Type }\}
\end{array}
\end{array}
$$

A simple type is composed from atomic types by choice or occurrence. Every simple type is a type.

$$
\begin{aligned}
\text { SimpleType }:= & \text { AtomicTypeName } \\
& \mid \begin{array}{l}
\text { SimpleType I SimpleType } \\
\text { SimpleType Occurrence }
\end{array}
\end{aligned}
$$

We saw many examples of types and simple types in Section 2.

\subsection{Top level definitions}

At the top level, one can define elements, and types.

$$
\begin{aligned}
\text { Definition }:=\text { define element ElementName TypeSpecifier } & \\
\mid & \text { define type TypeName TypeDerivation }
\end{aligned}
$$

Global element declarations, like local element declarations, consist of a name and a type specifier. A global type declaration specifies both the derivation and the declared type. We saw many examples of definitions in Section 2.

\subsection{Built-in type declarations}

The two XML Schema built-in types xs:anyType and xs:anySimpleType are defined as follows. 


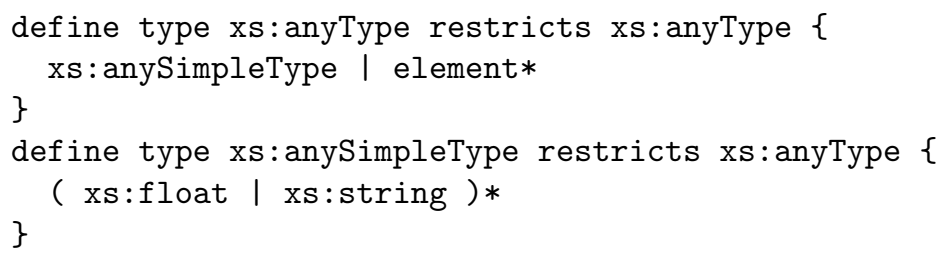

\section{Relationships between names}

We need auxiliary judgments to describe relationships between element names and between type names.

\subsection{Element name sets}

An element name set is either a singleton consisting of just the given element name, or the wildcard $*$ describing the set of all element names.

$$
\text { ElementNameSet }::=\text { ElementName } \mid *
$$

The judgment

ElementName within ElementNameSet

holds when the element name is within the specified element name set. For example:

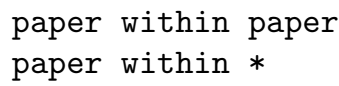

An element name is within the set consisting of just that element name.

\section{ElementName within ElementName}

An element name is within the set consisting of all element names.

$$
\overline{\text { ElementName within * }}
$$

\subsection{Derives}

The judgment

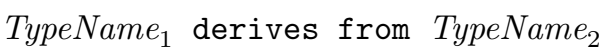

holds when the first type name derives from the second type name. For example, 


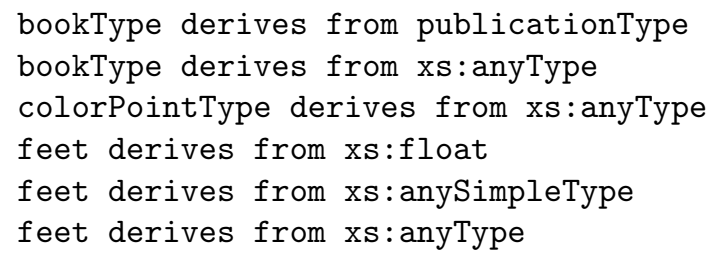

This relation is a partial order: it is reflexive and transitive by the rules below, and it is asymmetric because no cycles are allowed in derivation by restriction or extension.

Derivation is reflexive and transitive.

$$
\begin{aligned}
& \text { TypeName derives from TypeName } \\
& \text { TypeName }_{1} \text { derives from TypeName } \\
& \text { TypeName }_{2} \text { derives from TypeName } \\
& \hline \text { TypeName }_{1} \text { derives from TypeName }
\end{aligned}
$$

Every type name derives from the type it is declared to derive from by restriction or extension.

$$
\begin{gathered}
\frac{\text { define type TypeName restricts BaseTypeName }}{\text { TypeName derives from BaseTypeName }} \\
\frac{\text { define type TypeName restricts BaseTypeName }\{\text { Type }\}}{\text { TypeName derives from BaseTypeName }} \\
\frac{\text { define type TypeName extends BaseTypeName }\{\text { Type }\}}{\text { TypeName derives from BaseTypeName }}
\end{gathered}
$$

\section{$5 \quad$ Auxiliary judgments}

We now define two auxiliary judgments that are used in matching and validation. Here is the rule from matching that uses these judgments.

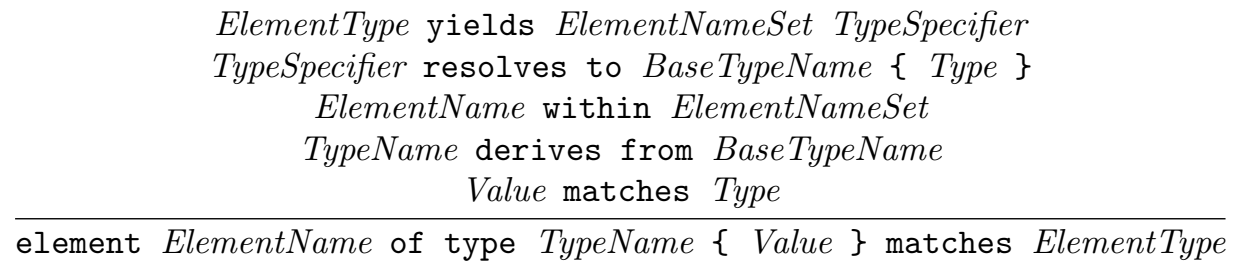

The element type yields an element name set and a type specifier, and the type specifier resolves to a base type name and a type. Then the given element matches the element type if three things hold: the element name must be within the element name set, the type name must derive from the base type name, and the value must match the type. 


\subsection{Yields}

The judgment

\section{ElementType yields ElementNameSet TypeSpecifier}

takes an element type and yields an element name set and a type specifier. For example,

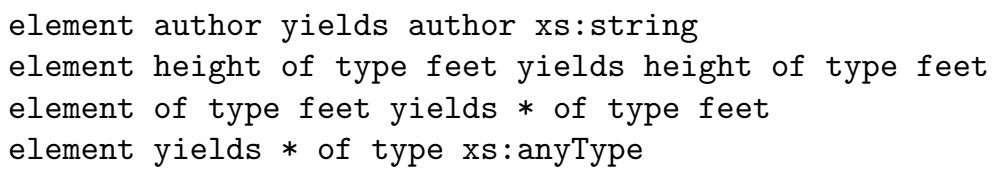

If the element type is a reference to a global element, then it yields the the name of the element and the type specifier from the element declaration.

$$
\frac{\text { define element ElementName TypeSpecifier }}{\text { element ElementName yields ElementName TypeSpecifier }}
$$

If the element type contains an element name and a type specifier, then it yields the given element name and type specifier.

$\overline{\text { element ElementName }\{\text { TypeSpecifier }\} \text { yields ElementName TypeSpecifier }}$

If the element type contains only a type specifier, then it yields the wildcard name and the type specifier.

$$
\overline{\text { element }\{\text { TypeSpecifier }\} \text { yields } * \text { TypeSpecifier }}
$$

If the element type has no element name and no type specifier, then it yields the wildcard name and the type xs: anyType.

$\overline{\text { element yields } * \text { xs:anyType }}$

\subsection{Resolution}

The judgment

$$
\text { TypeSpecifier resolves to TypeName \{ Type }\}
$$

resolves a type specifier to a type name and a type. For example,

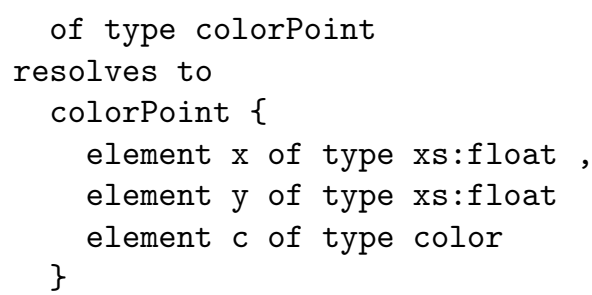


and

restricts xs:float resolves to xs:float \{xs:float $\}$

and

restricts publicationType \{

element author + ,

element title,

element year

\}

resolves to

publicationType \{

element author + ,

element title,

element year

\}

and

extends pointType \{ \}

element $c$ of type color

resolves to

colorPoint \{

element $x$ of type xs:float,

element $y$ of type xs:float

\}

element c of type color

If the type specifier references a global type, then resolve the type derivation in its definition, yielding a base type name and a type. Resolution returns the type name and the type (the base type name is discarded).

$$
\begin{aligned}
& \text { define type TypeName TypeDerivation } \\
& \text { TypeDerivation resolves to BaseTypeName \{ Type }\} \\
& \hline \text { of type TypeName resolves to TypeName }\{\text { Type }
\end{aligned}
$$

If the type specifier restricts an atomic type, then return the atomic type as both the type name and the type.

restricts AtomicTypeName
resolves to AtomicTypeName \{AtomicTypeName\}

If the type specifier is a restriction of a non-atomic type, then return the given type name and the given type.

restricts TypeName \{ Type \} resolves to TypeName \{ Type $\}$ 
If the type specifier is an extension, then resolve the name to get the base type, and return the given type name, and the result of concatenating the base type and the given type.

$\frac{\text { of type TypeName resolves to TypeName }\{\text { BaseType }\}}{\text { extends TypeName }\{\text { Type }\} \text { resolves to TypeName }\{\text { BaseType, Type }\}}$

\section{Matches}

The judgment

$$
\text { Value matches Type }
$$

holds when the given value matches the given type. For example,

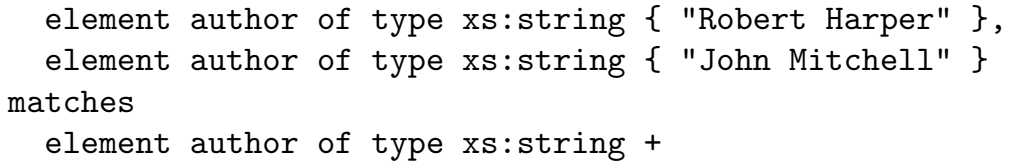

The empty sequence matches the empty sequence type.

$$
\text { () matches () }
$$

If two values match two types, then their sequence matches the corresponding sequence type.

Value $_{1}$ matches Type
Value $_{2}$ matches Type
$\frac{\text { Value }_{1}, \text { Value }_{2} \text { matches Type }}{1}$, Type $_{2}$

If a value matches a type, then it also matches a choice type where that type is one of the choices. 


$$
\begin{aligned}
& \text { Value matches } \text { Type }_{1} \\
& \overline{\text { Value matches } \text { Type }_{1} \text { । Type }} \text { T }_{2} \\
& \text { Value matches } \text { Type }_{2} \\
& \overline{\text { Value matches } \text { Type }_{1} \text { | Type }}
\end{aligned}
$$

A value matches an optional occurrence of a type if it matches either the empty sequence or the type.

$$
\frac{\text { Value matches () I Type }}{\text { Value matches Type? }}
$$

A value matches one or more occurrences of a type if it matches a sequence of the type followed by zero or more occurrences of the type.

$$
\frac{\text { Value matches Type, Type* }}{\text { Value matches Type+ }}
$$

A value matches zero or more occurrences of a type if it matches an optional one or more occurrences of the type.

$$
\frac{\text { Value matches Type+? }}{\text { Value matches Type* }}
$$

A string matches an atomic type name if the atomic type name derives from xs:string. Similarly for floats.

$$
\begin{aligned}
& \frac{\text { AtomicTypeName derives from xs:string }}{\text { String matches AtomicTypeName }} \\
& \frac{\text { AtomicTypeName derives from xs:float }}{\text { Float matches AtomicTypeName }}
\end{aligned}
$$

The rule for matching elements was explained at the beginning of Section 5 .

$$
\begin{gathered}
\text { ElementType yields ElementNameSet TypeSpecifier } \\
\text { TypeSpecifier resolves to BaseTypeName \{ Type }\} \\
\text { ElementName within ElementNameSet } \\
\text { TypeName derives from BaseTypeName } \\
\text { Value matches Type } \\
\hline \text { element ElementNameof type TypeName }\{\text { Value }\} \text { matches ElementType }
\end{gathered}
$$




\section{$7 \quad$ Erasure}

\subsection{Simply erases}

To define erasure, we need an ancillary judgment. The judgment

$$
\text { SimpleValue simply erases to String }
$$

holds when SimpleValueerases to the string String. For example,

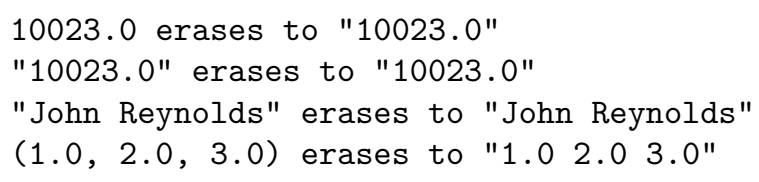

The empty sequence erases to the empty string.

$$
\text { () simply erases to "" }
$$

The concatenation of two non-empty sequences of values erases to the concatenation of their erasures with a separating space.

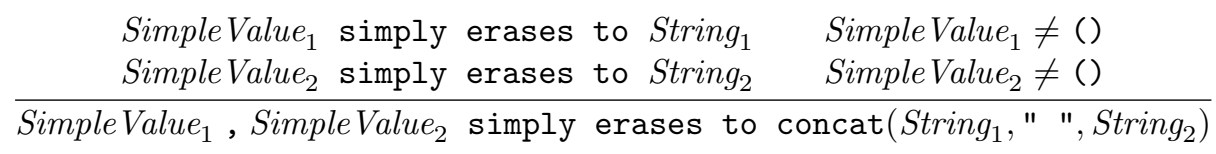

A string erases to itself.

$$
\overline{\text { String simply erases to String }}
$$

A float erases to any string that represents it.

$\overline{\text { float-of-string(String) simply erases to String }}$

\section{$7.2 \quad$ Erases}

The judgment

$$
\text { Value erases to UntypedValue }
$$

holds when the given value erases to the untyped value. For example,

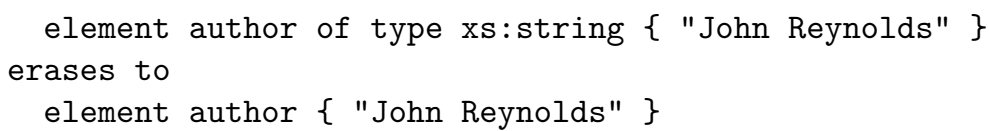

and 


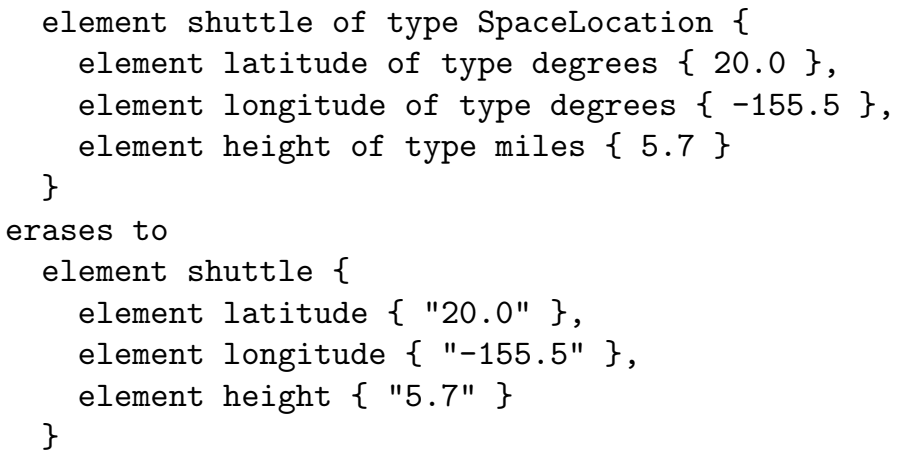

The empty sequence erases to itself.

$$
\overline{\text { () erases to () }}
$$

The erasure of the concatenation of two values is the concatenation of their erasure, so long as neither of the two original values is simple.

$$
\begin{gathered}
\text { Value }_{1} \text { erases to }{\text { Untyped } \text { Value }_{1}}_{\text {Value }} \text { not a simple value } \\
\text { Value }_{2} \text { erases to UntypedValue } \\
\text { Value }_{1}, \text { Value }_{2} \text { erases to UntypedValue }
\end{gathered}
$$

The erasure of a simple value is the corresponding string content using simpler erasure.

$$
\frac{\text { SimpleValue simply erases to String }}{\text { SimpleValue erases to String }}
$$

The erasure of an element is an element that has the same name and the erasure of the given content.

$$
\begin{aligned}
& \text { Value erases to UntypedValue } \\
& \text { element ElementName of type TypeName }\{\text { Value }\} \\
& \text { erases to } \\
& \text { element ElementName }\{\text { UntypedValue }\}
\end{aligned}
$$

\section{Validation}

\subsection{Simply validate}

The judgment

simply validate as SimpleType $\{$ String $\} \Rightarrow>$ SimpleValue

holds if validating the string against the simple type succeeds and returns the simple value. For example, 
simply validate as $x \mathrm{~s}:$ float $\{" 10023.0 "\} \Rightarrow 10023.0$

simply validate as xs:string $\{$ "10023.0" $\} \Rightarrow>10023.0 "$

simply validate as xs:string $\{$ "John Reynolds" $\} \Rightarrow$ "John Reynolds"

simply validate as $x$ :float* $\{\| 1.02 .03 .0 "\} \Rightarrow(1.0,2.0,3.0)$

Simply validating a string against a choice type yields the result of simply validating the string against either the first or second type in the choice.

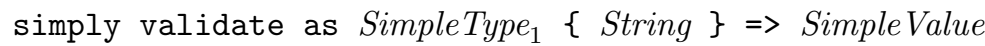

simply validate as SimpleType $_{1} \mid$ SimpleType $_{2}\{$ String $\} \Rightarrow$ SimpleValue

The rules for occurrences look slightly different from those in matching, because the simple types do not include the empty sequence or sequencing. Validating one or more occurrences breaks into two cases. In the first there is exactly one occurrence; in the second there is one occurrence followed by one or more occurrences, where the strings are separated by a space.

$$
\begin{aligned}
& \text { simply validate as SimpleType? }\{" \|\} \Rightarrow() \\
& \text { simply validate as SimpleType }\{\text { String }\} \Rightarrow>\text { SimpleValue } \\
& \text { simply validate as SimpleType? }\{\text { String }\} \Rightarrow \text { SimpleValue } \\
& \text { simply validate as SimpleType }\{\text { String }\} \Rightarrow>\text { SimpleValue } \\
& \text { simply validate as SimpleType+ }\{\text { String }\} \Rightarrow \text { SimpleValue } \\
& \text { simply validate as SimpleType }\left\{\text { String }_{1}\right\} \Rightarrow \text { SimpleValue }_{1}
\end{aligned}
$$

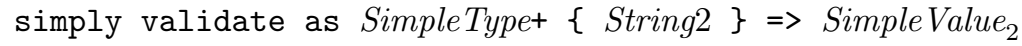

$$
\begin{aligned}
& \text { simply validate as SimpleTypet }\left\{\operatorname{concat}\left(\text { String }_{1}, " \text { ", } \text { String }_{2}\right)\right\} \\
& =>\text { Simple Value }_{1} \text {, Simple Value } 2 \\
& \text { simply validate as SimpleType+? }\{\text { String }\} \Rightarrow>\text { SimpleValue } \\
& \text { simply validate as SimpleType* }\{\text { String }\} \Rightarrow \text { SimpleValue }
\end{aligned}
$$

Simply validating a string against an atomic type derived from xs:string yields the string itself.

$$
\frac{\text { AtomicTypeName derives from xs:string }}{\text { simply validate as AtomicTypeName }\{\text { String }\} \Rightarrow \text { String }}
$$

Simply validating a string against an atomic type derived from xs:float yields the result of converting the string to a float.

$$
\begin{gathered}
\text { AtomicTypeName derives from xs:float } \\
\text { simply validate as AtomicTypeName }\{\text { String }\} \\
\Rightarrow>\text { float-of-string }(\text { String })
\end{gathered}
$$




\subsection{Validate}

The judgment

validate as Type $\{$ UntypedValue $\} \Rightarrow>$ Value

holds if validating the untyped value against the type succeeds and returns the value. For example,

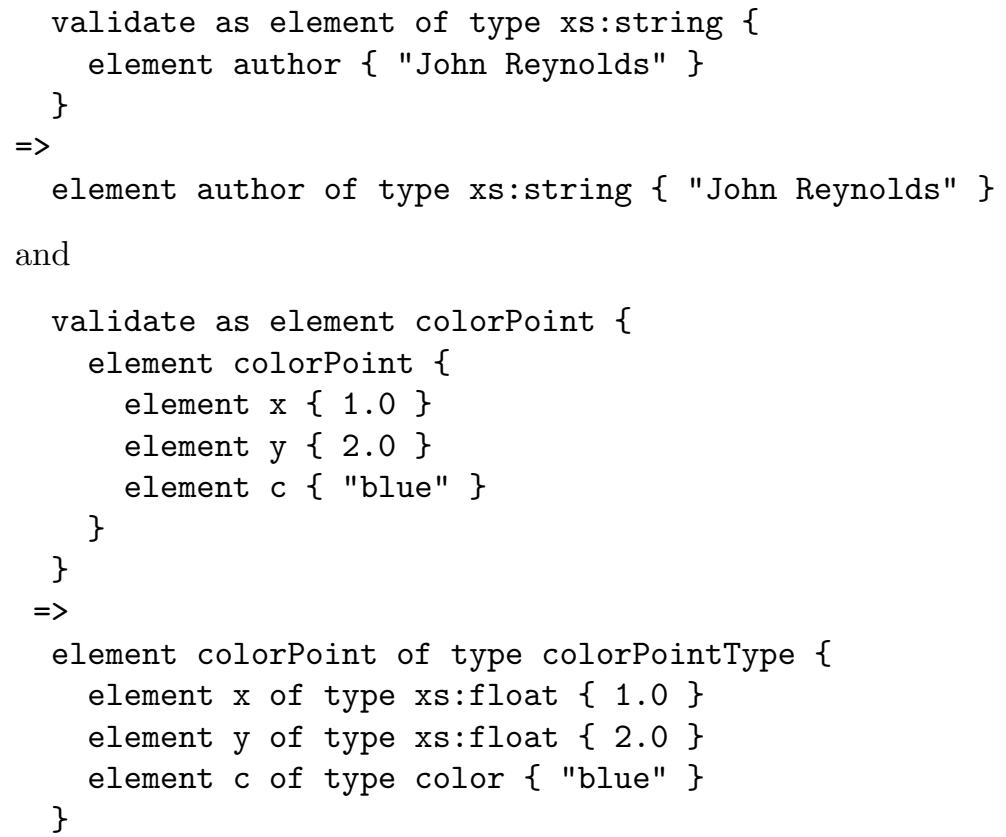

Validating the empty sequence as the empty type yields the empty sequence.

$$
\overline{\text { validate as () }\{()\} \Rightarrow()}
$$

Validating a concatenation of untyped values against a concatenation of types yields the concatenation of the validated values.

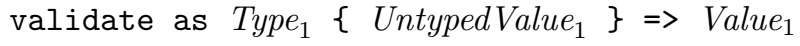

$$
\begin{aligned}
& \text { validate as } \text { Type }_{2}\left\{\text { UntypedValue }{ }_{2}\right\} \Rightarrow \text { Value }_{2} \\
& \overline{\text { validate as } \text { Type }_{1}, \text { Type }_{2}\left\{\text { UntypedValue }_{1}, \text { UntypedValue }_{2}\right\}} \\
& \Rightarrow \text { Value }_{1}, \text { Value }_{2}
\end{aligned}
$$

Validating a value against a choice type yields the result of validating the value as either the first or second type in the choice.

$$
\frac{\text { validate as } \text { Type }_{1}\{\text { UntypedValue }\} \Rightarrow>\text { Value }}{\text { validate as } \text { Type }_{1} \mid \text { Type }_{2}\{\text { UntypedValue }\} \Rightarrow \text { Value }}
$$




$\frac{\text { validate as } \text { Type }_{2}\{\text { UntypedValue }\} \Rightarrow \text { Value }}{\text { validate as } \text { Type }_{1} \mid \text { Type }_{2}\{\text { UntypedValue }\} \Rightarrow \text { Value }}$

The validation rules for occurrences are similar to the rules for occurrences in matching.

$$
\begin{gathered}
\frac{\text { validate as }(() \mid \text { Type })\{\text { UntypedValue }\} \Rightarrow \text { Value }}{\text { validate as Type? }\{\text { UntypedValue }\} \Rightarrow>\text { Value }} \\
\frac{\text { validate as (Type, Type* })\{\text { UntypedValue }\} \Rightarrow \text { Value }}{\text { validate as Type }\{\text { UntypedValue }\} \Rightarrow>\text { Value }} \\
\frac{\text { validate as Type }+\{\text { UntypedValue }\} \Rightarrow \text { Value }}{\text { validate as Type* }\{\text { UntypedValue }\} \Rightarrow \text { Value }}
\end{gathered}
$$

Validating a string against a simple type is defined in the previous section.

$$
\frac{\text { simply validate as SimpleType }\{\text { String }\} \Rightarrow>\text { SimpleValue }}{\text { validate as SimpleType }\{\text { String }\}=>\text { SimpleValue }}
$$

Validating an element against an element type is described by the following rule.

$$
\begin{gathered}
\text { ElementType yields ElementNameSet TypeSpecifier } \\
\text { TypeSpecifier resolves to BaseTypeName }\{\text { Type }\} \\
\text { ElementName within ElementNameSet } \\
\text { validate as Type }\{\text { UntypedValue }\}=>\text { Value } \\
\hline \text { validate as ElementType }\{\text { element ElementName }\{\text { UntypedValue }\}\} \\
\Rightarrow>\text { element ElementName of type BaseTypeName }\{\text { Value }\}
\end{gathered}
$$

The element type yields an element name set and a type specifier, and the type specifier resolves to a base type name and a type. Then the given element matches the element type if two things hold: the element name must be within the element name set, and validating the untyped value against the type must yield a value. The resulting element has the element name, the base type name, and the validated value.

\section{Ambiguity and the validation theorem}

For a given type, validation takes an external representation (an untyped value) into an internal representation (a value annotated with types). For a given type, we would like each external representation to correspond to just one internal representation, and conversely. We show that this is the case if the type is unambiguous, using a characterization of validation in terms of erasure and matching. 
Ambiguity. Validation is a judgment that relates a type and an untyped value to a value.

$$
\text { validate as Type }\{\text { UntypedValue }\} \Rightarrow \text { Value }
$$

In most of the examples we have seen, validation behaves as a function. That is, for a given type, for every untyped value, there is at most one value such that the above judgment holds. In this case, we say the type is unambiguous. But just as there is more than one way to skin a cat, sometimes there is more than one way to validate a value.

Here is an example of an ambiguous complex type:

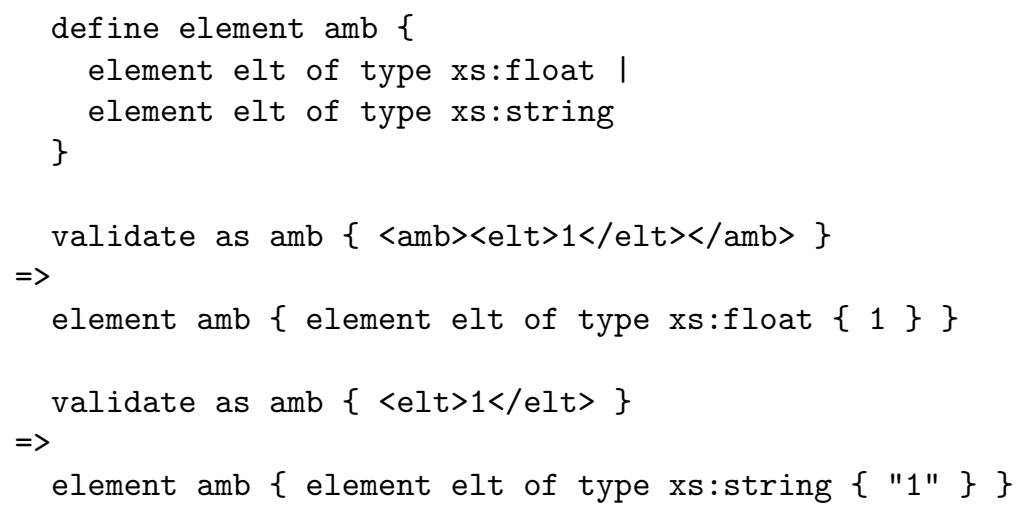

Here is an example of an ambiguous simple type:

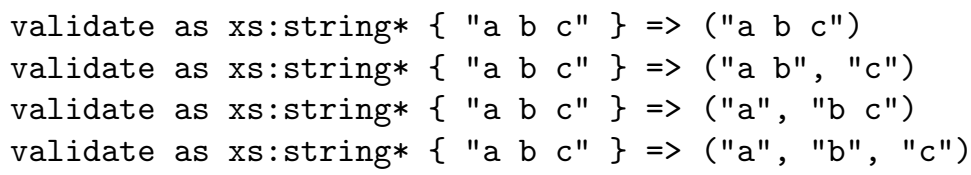

There are well known algorithms for determining when regular expressions are ambiguous, and there are similar algorithms for regular tree grammars $[12$, 7]. These are easily adapted to give an algorithm for determining when a given type is ambiguous.

In Schema, the issue of ambiguity is resolved differently than here. Complex types are required to be unambiguous. Simple types have rules that resolve the ambiguity: every space is taken as a list separator, and in a union the first alternative that matches is chosen. Thus, for the first example above Schema deems the type illegal, while for the second example above Schema validation yields the last of the four possibilities.

Our formal model differs from Schema for two reasons. First, while Schema is concerned solely with validation against types written by a user, XQuery must also support type inference. And while it may be reasonable to require that a user write types that are unambiguous, it is not reasonable to place this restriction on a type inference system. For example, if expression $e_{0}$ has type $\mathrm{xs}$ : boolean 
and $e_{1}$ has type $t_{1}$ and $e_{2}$ has type $t_{2}$, the expression if $\left(e_{0}\right)$ then $e_{1}$ else $e_{2}$ has type $t_{1} \mid t_{2}$, and it is not reasonable to require that $t_{1}$ and $t_{2}$ be disjoint.

Second, defining validation as a relation rather than a function permits a simple characterization of validation in terms of matching and erasure, as given in the next section.

The validation theorem. We can characterize validation in terms of erasure and matching.

Theorem 1. We have that

validate as Type $\{$ UntypedValue $\} \Rightarrow$ Value

if and only if

Value matches Type

and

Value erases to UntypedValue

The proof is by induction over derivations.

We would like to know that if we convert an external value to an internal value (using validation) and then convert the internal value back to an external value (using erasure) that we end up back where we started. This follows immediately from the validation theorem.

Corollary 1. If

validate as Type $\{$ UntypedValue $\} \Rightarrow$ Value and

Value erases to Untyped Value' then

UntypedValue $=$ UntypedValue

Proof. From the first hypothesis and the validation theorem we have that Value erases to UntypedValue

Taking this together with the second hypothesis and the fact that erasure is a function, the conclusion follows immediately.

Similarly, we would like to know that if we convert an internal value of a given type to an external value (using erasure) and then convert the internal value back to an external value (using validation against that type) that we again end up back where we started, so long as the type is unambiguous. Again, this follows immediately from the validation theorem.

Corollary 2. If

Value matches Type

and

Value erases to UntypedValue and

validate as Type $\{$ UntypedValue $\} \Rightarrow$ Value $^{\prime}$ and 
Type is unambiguous

then

Value $=$ Value

Proof. By the validation theorem, we have that the first two hypotheses are equivalent to

validate as Type $\{$ UntypedValue $\} \Rightarrow$ Value

Taking this together with the third hypothesis and the fact that validate is a function when the type is unambiguous, the conclusion follows immediately.

\section{References}

1. Tim Bray, Jean Paoli, and C. M. Sperberg-McQueen. Extensible Markup Language (XML) 1.0. W3C Recommendation, February 1998.

2. Allen Brown, Matthew Fuchs, Jonathan Robie, and Philip Wadler. MSL - a model for W3C XML Schema. In Proceedings of International World Wide Web Conference, pages 191-200, Hong Kong, China, 2001.

3. James Clarke. XSL Transformations (XSLT) version 1.0. W3C Proposed Recommendation, October 1999.

4. James Clarke. TREX - Tree Regular Expressions for XML. Thai Open Source Software Center, February 2001.

5. James Clarke and Murata Makoto. RELAX NG specification. Oasis, December 2001.

6. Sophie Cluet, Claude Delobel, Jérôme Siméon, and Katarzyna Smaga. Your mediators need data conversion! In Proceedings of ACM Conference on Management of Data (SIGMOD), pages 177-188, Seattle, Washington, June 1998.

7. H. Comon, M. Dauchet, R. Gilleron, F. Jacquemard, D. Lugiez, S. Tison, and M. Tommasi. Tree automata techniques and applications, 1997.

8. Haruo Hosoya and Benjamin C. Pierce. XDuce: an XML processing language. In International Workshop on the Web and Databases (WebDB'2000), Dallas, Texas, May 2000.

9. Michael Kay. XSL Transformations (XSLT) version 2.0. W3C Working Draft, April 2002.

10. Murata Makoto. Document description and processing languages - regular language description for XML (relax), October 2000.

11. Yannis Papakonstantinou and Victor Vianu. DTD inference for views of XML data. In Proceedings of ACM Symposium on Principles of Database Systems (PODS), Dallas, Texas, May 2000.

12. Grzegorz Rozenberg and Arto Salomaa, editors. Handbook of Formal Languages. Springer-Verlag, 1997.

13. Henri S. Thompson, David Beech, Murray Maloney, and N. Mendelsohn. XML Schema part 1: Structures. W3C Recommendation, May 2001.

14. XPath 2.0. W3C Working Draft, April 2002.

15. XQuery 1.0: An XML query language. W3C Working Draft, April 2002.

16. XQuery 1.0 formal semantics. W3C Working Draft, March 2002. 